\title{
Marie Nyswanders Beitrag: Der Drogenabhängige als Patient
}

\begin{abstract}
Zusammenfassung: Dieser Artikel schildert die besonderen Verdienste Mary Nyswanders in der Betrachtungsweise der Behandlung von Drogenabhängigen. Dabei spielte die Charakterisierung des Drogenabhängigen als Patienten eine besondere Rolle, nämlich in der Methadon-Erhaltungsbehandlung, die auf eine dauerhafte Behandlung und nicht auf vollständige und sofortige Heilung abzielt.
\end{abstract}

Schlüsselwörter: Opiatabhängigkeit - Ethische Probleme Methadon - Substitutionsbehandlung - Patientenbild

The Contribution of Marie Nyswander - The Drug Addict as a Patient: This article describes the special contribution of Marie Nyswander's way of looking at the treatment of drug addicts. She focused on the characterization of the drug addict as a patient, namely in the methadone maintenance treatment, which aims at a long-lasting treatment and not at complete and immediate remission.

Key words: Opiate Addiction - Ethical Problems - Methadone - Substitution Maintenance - Patient Image

Am 15. Juni 1996 wurde in der Hansestadt Hamburg eine neue Straße eingeweiht - der Marie-Nyswander-Weg. Dieses Ereignis fand in einem feierlichen Rahmen statt: Es gab einen Umzug, es spielte eine Blaskapelle und es wurden Festansprachen gehalten. Dazu gab es ein Angebot an verschiedenen Speisen, die von den Anwohnern und den Klienten und Mitarbeitern der Palette zubereitet worden waren.

Ich hatte die große Freude und das Privileg, daran teilnehmen zu können, zusammen mit Marie Nyswanders langjährigem Lebenspartner und Arbeitskollegen, Professor Dr. Vincent Dole. Maries Mutter, Professor Dr. Dorothy Nyswander, die damals 102 Jahre alt war und in Kalifornien lebte, konnte leider nicht persönlich erscheinen; es waren aber viele ihrer Freunde anwesend. Sie teilte mir später mit, dass die Namensgebung dieser Hamburger Straße zu Ehren ihres einzigen Kindes das schönste Erlebnis in ihrem langen Leben gewesen sei.

Suchttherapie Sonderheft 2001; 2: S11-S14 (c) Georg Thieme Verlag Stuttgart · New York ISSN 1439-9903
Robert G. Newman

Beth Israel Medical Center, New York, USA
Ein großartiger Kollege, Josh von Soer Clemm von Hohenberg, der gebürtig aus den Niederlanden stammte, aber jahrelang in Hamburg lebte und auch dort arbeitete, hatte sein Leben der Hilfe all derer und ihrer Freunde und Angehörigen gewidmet, die von Abhängigkeitserkrankungen betroffen sind. Ich muss gestehen, ich war sprachlos, als mich Josh damals anrief und mir mitteilte, dass seine jahrelangen eigenhändigen Bemühungen, Marie (die zehn Jahre zuvor verstorben war) mit einer nach ihr zu Ehren benannten Straße Anerkennung zu zollen, schließlich von all den vielen dafür zuständigen Behörden genehmigt worden war.

Nirgends sonst auf der Welt gibt es eine vergleichbare Anerkennung, weder für Marie noch für Vince Dole - nicht in den USA, wo sie ihre Pionierarbeit leisteten und wo heute über 160000 Menschen Methadonbehandlungen erhalten; nicht in Hongkong, wo Heroinabhängigen seit über einem Vierteljahrhundert ein sofortiger Zugang (d.h. Behandlungsbeginn noch am Tage der Anmeldung) zur Methadonbehandlung gewährt wird (und wo es - nicht rein zufällig - eine der niedrigsten HIV-Prävalenzraten bei intravenösen Drogengebrauchern gibt); nicht in England; nicht in Australien, Frankreich und auch nicht in Kirgistan, Litauen, Mazedonien oder anderen Ländern, die Methadon in den vergangenen Jahren als Reaktion auf eine große AIDS-Epidemie, Hepatitis C und anderen mit intravenösem Drogengebrauch assoziierten Erkrankungen einführten.

Deutschland ist nicht nur die einzige Nation, die Marie Nyswander auf eine derart deutliche Weise geehrt hat, es ist auch einer der letzten Orte, für die ich es vorhergesagt hätte, dass sie einmal einem Gründer der Methadonbehandlung eine solche Ehre erweisen würden. Schließlich hatte, noch weniger als 10 Jahre zuvor und gerade einmal fünf Jahre, bevor Josh seine Kampagne begann, der damalige nordrheinwestfälische Gesundheitsminister Hermann Heinemann korrekterweise Methadon als ein „Tabu-Wort“ bezeichnet [1] und der Berliner Drogenbeauftragte veröffentlichte gar einen Artikel mit dem aufschlussreichen Titel „Warum es in Deutschland kein Methadonprogramm geben wird“ [2]!

Josh hat Marie nie getroffen. Er hat aber die wahrhaft bemerkenswerten Veränderungen, die Methadonbehandlungen im Leben all derjenigen bewirken können, die bis vor kurzem noch Sklaven ihrer Abhängigkeit waren und denen es bisher an Hilfe und Hoffnung mangelte, miterleben dürfen: nämlich wieder ein sozial produktives, gesundes und persönlich lohnenswertes Leben führen zu können. 
Nebenbei bemerkt, um Ihnen einen Hinweis darauf zu geben, was für ein außergewöhnlich großzügiger und lieber Mensch Josh war (er verstarb leider im März 2000 an Krebs): Er unternahm einmal einen „kurzen Abstecher“ von über tausend Meilen von einem Meeting in Arizona nach Berkeley in Kalifornien, um Dr. Dorothy Nyswander persönlich das riesige und schwere metallene Straßenschild zu überreichen, dass ihrer Tochter Marie Anerkennung zollt. Dieses Schild hing seitdem über ihrem Bettende und bereitete der bettlägerigen Dame täglich Freude, bis sie im Dezember 1998, im Alter von 104 Jahren, verstarb.

Sie können sich sicher vorstellen, dass ich der Marie zuteil gewordenen Ehre mit größter Begeisterung und ohne jeglichen Vorbehalt zustimme. Aber ich erachte es als äußerst wichtig, unsere Aufmerksamkeit auf genau das zu richten, was ihre Leistung so einmalig machte - was so revolutionär daran war, die Einstellung gegenüber Drogenabhängigkeit und die Behandlung von Abhängigen zu verändern.

- Es war nicht die Entdeckung des synthetischen Opioids Methadon. Dieses Verdienst gebührt deutschen Wissenschaftlern, die es bereits 1939 im Rahmen der Schmerzmittelforschung als Alternative zum Opiumderivat Morphin synthetisiert hatten [3].

- Dr. Nyswander trug auch nicht dazu bei, Methadon in der medikamentösen Behandlung Drogenabhängiger einzusetzen. Bereits im Jahre 1947 wurden in Amerika erste Studien zur Anwendungsmöglichkeit des Medikamentes in diesem Einsatzgebiet vorgelegt [4].

- Es war auch nicht die Konzeption der Methadon-Erhaltungsbehandlung (,methadone maintenance“). Bereits einige Jahre vor der ersten Veröffentlichung über diesen Behandlungsansatz durch die Doktoren Dole und Nyswander schrieb ein kanadischer Arzt, Robert Halliday, einen Artikel, in dem er Folgendes anführte: „Ein Arzt darf Narkotika oder andere Drogen zum Zwecke der Behandlung eines Drogenabhängigen einsetzen, vorausgesetzt, dass diese Behandlung Teil eines umfassenden Therapiekonzeptes ist [5].“ Etwas später veröffentlichte Dr. Halliday mit seiner Kollegin, Ingeborg Paulus, einen Erfahrungsbericht der bis in das Jahr 1959 zurückreichte! - über Patienten, die täglich Methadon über unbefristete Zeiträume erhalten hatten. Sie hoben besonders hervor: „Es ist nicht nur unlogisch, sondern auch naiv und irreführend zu fordern, dass Abstinenz das einzige Kriterium sein soll, nach dem eine Veränderung des Abhängigen beurteilt werden kann.“ Während sie einerseits eine fortgesetzte, unbefristete Erhaltungsmedikation mit Methadon guthießen, legten sie andererseits eine willkürliche Höchstgrenze der Tagesdosierung von $40 \mathrm{mg}$ fest und beharrten auf einem universalen Abstinenzziel: „Ziel ist es, den Patienten letztendlich von seiner Drogenabhängigkeit zu befreien ... [6]“

- Der revolutionäre Aspekt an der von Marie und Vince eingeführten Methadon-Erhaltungsbehandlung hatte aber weder etwas mit der Dosierung des eingesetzten Medikamentes noch mit der Behandlungsdauer zu tun. Während sie berichteten, dass ihre ursprünglichen Patienten zwar zwischen 50 und $150 \mathrm{mg}$ pro Tag erhielten, lehnten sie jedoch ein starres Festhalten an einer bestimmten Dosis ab und warfen auch nicht die Frage auf, ob, wann und bei wem die Behandlung beendet werden sollte [7].
- Und was ihre „Metabolismustheorie“ anbelangt: Auch wenn diese angesichts der späteren Entdeckung von Endorphin-Rezeptoren und anderer physiologischer Mechanismen aus heutiger Sicht zwar als „voraussagende Theorie" bewertet werden könnte, so war sie dennoch nur ein Vorschlag bzw. ein Versuch, um die von ihnen beobachtete außergewöhnliche Reaktion auf die Methadonbehandlung und die in scharfem Gegensatz dazu stehenden, von ihnen und anderen gesammelten Erfahrungen und Ergebnisse mit anderen Behandlungsformen erklären zu können [8].

Und so steht immer noch die Frage im Raum: Was war denn nun eigentlich Maries besonderes Verdienst? Bevor ich diese Frage beantworte, lassen Sie mich zuvor eine weitere verwandte Frage stellen: Ist die Suchtbehandlung mit Methadon erfolgreich? Dies ist eine Frage, auf die es mehr als nur eine Antwort gibt, so dass Kritiker und auch Befürworter gleichermaßen eine ihrem Geschmack entsprechende Antwort finden können! Insbesondere,

- Methadon ist ein schwerer Misserfolg - wenn das Behandlungsziel die „Heilung“ der Abhängigkeitserkrankung ist.

- Methadon ist ein beispielloser Erfolg - wenn es das Ziel ist, die Patienten zu einem normalen, produktiven und selbstverwirklichenden Leben befähigen zu können.

Aus diesen Antworten ergibt sich, dass die Wahrnehmung von Erfolg oder Misserfolg der Methadonbehandlung eine Funktion der angestrebten therapeutischen Ziele ist - und das sollte uns nicht überraschen, denn dies gilt für jede Form medizinischer Hilfeleistung. Stellen Sie sich doch nur einmal vor, man versuchte die "Heilungsrate“ im Rahmen der Behandlung von Schizophrenie, verschiedenen depressiven Erkrankungen oder dem Aufmerksamkeitsdefizitsyndrom (wogegen geschätzte 3 Millionen amerikanische Schulkinder Medikamente verabreicht bekommen) [9] oder irgendeiner anderen der vielen Erscheinungsformen psychopathologischer Krankheitsbilder zu messen.

Das Gleiche gilt auch für physische Krankheiten. Wir messen und beurteilen Erfolg in der Behandlung der meisten Krebsformen eher an Überlebensraten denn an „Heilungen“.

Die meisten Arten von schwerem Schmerz werden (oder besser sollten) mit Analgetika behandelt (werden), deren Zweck es ist, die Beschwerden zu beheben - und nicht die zugrunde liegende Pathologie umzukehren. Und Antiepileptika werden verschrieben, um Anfälle zu verhindern, und nicht, um deren Ursache(n) zu beseitigen. Tatsächlich ist es doch so, mit Ausnahme von einigen Antibiotika, dass man lange und intensiv überlegen muss, um ein Beispiel für eine medizinische Behandlung zu finden, die eine Heilung anstrebt oder gar auf eine Heilung hoffen lässt.

Trotz dieser entmutigenden Perspektive wird diese TherapieEinschränkung gleichermaßen von Patienten und Ärzten mit relativer Gelassenheit akzeptiert. Aber es gibt auch eine Ausnahme: die Behandlung von Abhängigkeitserkrankungen! Hier ist die generelle Regel unter Klinikern wie auch in der Bevölkerung als Ganzes, jeden therapeutischen Ansatz, dessen Ziel nicht „Heilung“ ist (definiert als das Erreichen dauerhafter Abstinenz), strikt abzulehnen. Dieses Ziel ist nicht nur einmalig im Bereich medizinischer Behandlung von Krankhei- 
ten, es widerspricht auch jeglichen logischen Erfahrungen, die seit vielen Jahrzehnten aus allen Teilen der Welt berichtet werden.

Sicherlich gibt es gelegentlich unerwartete Ausnahmen von der Regel, aber die große Mehrheit der Patienten erlebt schließlich einen Rückfall in den Drogengebrauch, ganz egal, welcher Art von Behandlung sie sich unterzogen haben (falls überhaupt der Fall), um Abstinenz zu erreichen. Des Weiteren muss man sich vor Augen führen, dass selbst wenn ein Abhängiger es irgendwie schaffte, die Ursache(n) der pathognonomischen Charakteristik der Abhängigkeitserkrankung das Rückfallpotenzial [10] - zu überwinden, es nicht möglich wäre, dies zu erkennen! Wer verkündet, dass ein Individuum „geheilt“" ist, ist ein Narr oder Scharlatan.

Opiatabhängigkeit ist vergleichbar mit der Alkoholkrankheit. In beiden Fällen steht die Ätiologie im Spektrum von Spekulationen und Theorien, von denen einige plausibler erscheinen mögen als andere, denen aber allen eine solide wissenschaftliche Grundlage fehlt. Wie bei jeder medizinischen Erkrankung sind vermutlich viele Faktoren beteiligt - genetische, physiologische, psychologische, soziale usw. Angesichts dieser mitwirkenden vielfältigen, komplexen und in Wechselbeziehung zueinander stehenden Ursachen, die nicht einmal mit Sicherheit identifiziert werden können, wundert es kaum, dass sie nicht behoben und ihre Einflüsse nicht rückgängig gemacht werden können.

Im Gegensatz zu den üblichen Einstellungen gegenüber Suchterkrankungen gibt es eine breite Akzeptanz der Grenzen (aber auch der Würdigung des großen Potenzials!) der Behandlung des Alkoholismus. Die Anonymen Alkoholiker (AA) zum Beispiel sind die am meisten respektierte und einflussreichste Stimme in diesem Sektor und sie haben vielen hunderttausenden von schweren Problemtrinkern dabei geholfen, eine Abstinenz zu erreichen und diese aufrechterhalten zu können. Und dennoch fußt das zentrale Prinzip der AAs auf der Annahme, dass Alkoholismus eine Krankheit ist, die nie jemals geheilt werden kann. Schon der kleinste und mit Bedacht formulierte Hinweis, dass Ausnahmen von dieser Regel existieren könnten, wird strikt als Ketzerei von der Hand gewiesen. Selbst nach vielen Jahrzehnten einer völligen Abstinenz von Alkohol sprechen AA-Anhänger noch von „genesenden Alkoholikern“ oder „abstinenten Alkoholikern“, aber niemals von „geheilten Alkoholikern“. Es scheint doch vernünftig zu sein anzunehmen, dass genau die gleiche Sichtweise auch auf Opiatabhängige zutrifft.

Aber wie ist es angesichts dieser nihilistischen Blickweise auf „Heilung“ möglich, dass es eine positive Antwort auf die Frage nach der Effizienz von Methadon in der Suchtbehandlung gibt? Die recht einfache Erklärung ist, dass man für diese Krankheit den gleichen Bewertungsmaßstab ansetzt wie für fast alle anderen Erkrankungen auch - nämlich das Ausmaß, in dem die Behandlung Patienten hilft, gesünder zu leben, weniger wahrscheinlich an ihrer Erkrankung oder deren Begleiterscheinungen $\mathrm{zu}$ sterben, und inwieweit sie in die Lage versetzt werden, ihr Schicksal selbst in die Hand nehmen zu können. Überträgt man diesen Maßstab auf die MethadonErhaltungsbehandlung, ist sie in der Tat eine äußerst wirksame Behandlungsform!
Und nun zurück zu meiner Eingangsfrage: Was ist das besondere Verdienst von Marie Nyswander, das die Dankbarkeit der Menschen überall auf der Welt rechtfertigt und wie sie am dramatischsten durch die Einweihung des MarieNyswander-Weges in der Hansestadt Hamburg zum Ausdruck kommt?

Ich denke, die Antwort findet sich in einem 1956 von ihr verfassten Buch - also fast ein Jahrzehnt vor dem ersten veröffentlichten Bericht zur Methadon-Erhaltungsbehandlung.

Der Titel sagt alles: The Drug Addict as a Patient [11]! - Der Drogenabhängige als Patient!

Dieses einfache Konzept ebnete den Weg, die Einstellung gegenüber und die Behandlung von Abhängigen zu revolutionieren - nämlich sie nicht als hedonistisch, amoralisch und als antisoziale Psychopathen zu begreifen, sondern als Patienten, die an einer Krankheit leiden, die von Ärzten behandelt werden kann und muss.

Dr. Nyswander erkannte (vor fast 50 Jahren!) die Torheit einer Null-Toleranz-Orientierung in Richtung einer völligen und dauerhaften Abstinenz und die Anwendung strafrechtlicher Sanktionen, um dieses Ziel zu erreichen. Sie äußerte sich folgendermaßen: „Trotz allerstrengster restriktiver Maßnahmen, die je in einem Land vollzogen wurden, um den Drogenhandel zu kontrollieren, zieht Drogenabhängigkeit auch nach 40 Jahren noch neue Rekruten heran, von denen viele noch sehr jung sind und denen eine lebenslange Flucht vor dem Gesetz bevorsteht ... Nur wenige Behörden glauben, dass die epidemischen Ausmaße auch nur spürbar reduziert worden sind [11]." Sie hob besonders die großen gesetzlichen Barrieren gegenüber medizinischer Behandlung hervor, die in Amerika errichtet wurden: „Ärzte sind nicht länger befugt, Abhängige zu behandeln ... [und deren Behandlung] liegt zum großen Teil in den Händen von Psychiatern [11].“

Vor dem Hintergrund ihrer eigenen Enttäuschungen mit dem Angebot traditioneller psychiatrischer Betreuung lehnte sie die weit verbreitete Meinung ab, dass Drogenrückfälle ausschließlich - oder sogar primär - durch zugrunde liegende psychologische Faktoren bedingt seien. „Obwohl Drogenabhängige wie andere Patienten mit medizinischen Krankheiten begleitende oder kausale emotionale Probleme haben, ist nicht gesagt, dass sie eine psychiatrische Hilfe benötigen oder diese wünschen [11].“

Bei der Beschreibung der Rolle, die Marie Ärzten im Bereich der Suchtbehandlung zudachte, war sie sprachgewandt und präzise: „Der Arzt ist in einer besonders günstigen Situation, um Drogenabhängigen zu helfen ... Dem Arzt muss es gelingen, ein ehrliches Verständnis für den Existenzkampf und die Schwierigkeiten des Patienten zu entwickeln und sich klarzumachen, dass sein einziges Interesse an der Sucht ein mit dem Patienten gemeinsames Interesse ist“, d.h., er fungiert nicht „nur“ als Mediziner, sondern auch als Befürworter seines Patienten [11].

Ebenso deutlich war sie in ihrer Ablehnung gegenüber „Angsteinflößung oder Anwendung von Drohungen“ im Rahmen der Suchtbehandlung, indem sie sagte: „Das ist die 
Aufgabe des Gesetzes, und es sollte nicht vom Arzt angewendet werden [11].“

Ungeachtet ihrer eigenen vergeblichen therapeutischen Bemühungen über viele Jahre hinweg schlug sie zum Ende ihres Buches einen von Entschlossenheit und Optimismus getragenen Ton an. Sie freute sich auf die Eröffnung „neuer Perspektiven für therapeutisches Experimentieren ... [und] die Erforschung von Therapieformen außerhalb der Atmosphäre von Strafanstalten." Sie fuhr fort mit der Prophezeiung: „Durch die Bestätigung, dass Sucht eine medizinische Erkrankung ist, ... kann man hoffen, einen entscheidenden historischen Wandel herbeizuführen, und Ärzte dazu ermutigen, dieses große medizinische und soziale Problem lösen zu helfen, so wie sie auch andere Probleme gelöst haben [11].“

Gerade einmal zehn Jahre später erwies sich ihr Optimismus als gerechtfertigt, in erster Linie als Folge ihrer eigenen beherzten und auf Wissenschaftlichkeit basierenden Arbeit, aber auch aufgrund ihres sich darin widerspiegelnden außergewöhnlichen Mitgefühls, gesunden Menschenverstandes und ihrer klinischen Fähigkeiten.

Natürlich war es Marie 1956 weder möglich, genau vorauszuahnen, in welcher Art und Weise sich der „historische Wandel“" vollziehen würde, noch erlaubte es ihr ihre große Bescheidenheit, den enormen Beitrag vorherzusagen, den sie selbst leisten würde, um diesen Wandel einzuleiten und $\mathrm{zu}$ vollenden. Und daher erlaube ich mir, ihn wie folgt zusammenzufassen: Es war, in einfachen Worten, ihre Einstellung gegenüber Abhängigen als Patienten! Sie ermutigte ihre Arztkollegen, Abhängige auf die gleiche Art und Weise zu betrachten wie alle anderen an ernsten, potenziell lebensbedrohlichen und chronischen Krankheiten leidenden Menschen und auch im Rahmen ihrer Behandlung die gleichen Zielsetzungen und Standards anzuwenden, die den Berufszweig der Medizin jahrtausendelang geleitet haben. Implizit enthält ihre Botschaft die Erkenntnis, dass, „Heilung“ im Allgemeinen nicht zu erzielen und schwer zu definieren ist, „Behandlung“ hingegen immer möglich ist. Und weil dies so ist, stellt die Behandlung für den Arzt sowohl eine Herausforderung als auch eine Verpflichtung dar.

Wir müssen alles Menschenmögliche tun, um die Hindernisse zu überwinden, die der Bereitstellung von verständnisvollen und wirksamen medizinischen Angeboten für Abhängige im Wege stehen. Dies erfordert Überredungskunst und eine umfassende Ausbildung sowie pädagogische Fähigkeiten, aber auch Beharrlichkeit - Fähigkeiten und Qualitäten, die Marie Nyswander reichlich besaß und die ihren phänomenalen Beitrag für das Wohlergehen von Abhängigen und deren Angehörigen überall auf der Welt ermöglichten.

Aber sie besaß noch eine weitere Eigenschaft, die vielleicht die wichtigste von allen war: Widerstandskraft all jenen gegenüber, die durch Einschüchterung und jedes andere verfügbare Mittel versuchten, die Arbeit zu blockieren, der gegenüber sie und Vince Dole sich verpflichtet fühlten! Wir müssen danach streben, ihrem Beispiel zu folgen!

\section{Danksagung}

Mein besonderer Dank gilt meinem Kollegen Ralf Gerlach, stellvertretender Leiter von INDRO e.V., für die Übersetzung meines Vortrages.

\section{Literatur}

${ }^{1}$ Heinemann H. Eröffnung des Symposiums. Medikamentengestützte Rehabilitation bei Drogenabhängigen. Düsseldorf: Ministerium für Arbeit, Gesundheit und Soziales, NRW, 1987

${ }^{2}$ Heckmann W. Warum es in Deutschland kein Methadonprogramm geben wird. Wiener Zeitschrift für Suchtforschung 1986; 9: $3-7$

${ }^{3}$ Bockmühl M, Ehrhart G. Spasmolytic and analgesic compounds. Annales de Chimie 1948; 561: 52-85

${ }^{4}$ Isbell H, Wikler A, Eddy NB et al. Tolerance and Addiction Liability of 6-dimethylamino-4-4-diphenyl-heptanone-3 (Methadone). Journal of the American Medical Association 1947; 135: 888-903

${ }^{5}$ Halliday R. Management of the Narcotic addict. British Columbia Medical Journal 1963; 5: 412-414

${ }^{6}$ Paulus I, Halliday R. Rehabilitation and the Narcotic Addict: Results of a comparative methadone withdrawal program. Canadian Medical Association Journal 1967; 96: 655-659

${ }^{7}$ Dole VP, Nyswander ME. A Medical Treatment for Diacetylmorphine (Heroin) Addiction - A Clinical Trial with Methadone Hydrochloride. Journal of the American Medical Association 1965; 193(8): 646-650

${ }^{8}$ Dole VP, Nyswander ME. Heroin Addiction - A Metabolic Disease. Archives of Internal Medicine 1967; 120: 19-24

${ }^{9}$ Kendrick C. Recreational Ritalin: A Disturbing Trend. San Francisco Chronicle. 18 March 2001

${ }^{10}$ Newman RG. The Need to Redefine Addiction. New England Journal of Medicine 1983; 308: 1096-1098

${ }^{11}$ Nyswander ME. The Drug Addict as a Patient. New York: Grune \& Stratton, 1956

Prof. MD Robert G. Newman

Beth Israel Medical Center

First Avenue at 16th Street

New York, NY 10663

USA

E-mail: rgnewmanmd@aol.com 